\title{
Somnography using unobtrusive motion sensors and Android-based mobile phones
}

\author{
Stefan Gradl $^{1}$, Heike Leutheuser ${ }^{1}$, Patrick Kugler ${ }^{1}$, Student Members, IEEE, EMBS, \\ Teresa Biermann ${ }^{2}$, Sebastian Kreil ${ }^{2}$, Johannes Kornhuber ${ }^{2}$, Matthias Bergner ${ }^{2}$ and \\ Bjoern Eskofier ${ }^{1}$, Member, IEEE, EMBS
}

\begin{abstract}
Sleep plays a fundamental role in the life of every human. The prevalence of sleep disorders has increased significantly, now affecting up to $50 \%$ of the general population. Sleep is usually analyzed by extracting a hypnogram containing sleep stages. The gold standard method polysomnography (PSG) requires subjects to stay overnight in a sleep laboratory and to wear a series of obtrusive devices. This work presents an easy to use method to perform somnography at home using unobtrusive motion sensors.

Ten healthy male subjects were recorded during two consecutive nights. Sensors from the Shimmer platform were placed in the bed to record accelerometer data, while reference hypnograms were collected using a SOMNOwatch system. A series of filters were used to extract a motion feature in $\mathbf{3 0}$ second epochs from the accelerometer signals. The feature was used together with the ground truth information to train a Naive Bayes classifiers that distinguished wakefulness, REM and nonREM sleep. Additionally the algorithm was implemented on an Android mobile phone.

Averaged over all subjects, the classifier had a mean accuracy of $79.0 \%$ (SD 9.2\%) for the three classes. The mobile phone implementation was able to run in realtime during all experiments. In future this will lead to a method for simple and unobtrusive somnography using mobile phones.
\end{abstract}

\section{INTRODUCTION}

Sleep plays a fundamental role in the life of every human. We spend about one third of our lifetime sleeping and the quality and quantity of sleep has a large impact on our physical and mental condition [1]. Some chronic diseases like diabetes, depression, and obesity can be associated with insufficient sleep [2]. The prevalence of sleep disorders has increased significantly, now affecting up to $50 \%$ of the general population [3]. Especially sleep deprivation can cause micro sleep or lapses in concentration which can lead to serious accidents [4].

Sleep is commonly divided into different phases with unique characteristics. Those phases are the same for all humans and correlate with the level of physical excitation. For example the deep sleep phase is usually associated with very low and regular brain and muscle activity, while REM (rapid eye movement) sleep corresponds to noticeably increased and irregular activity. Since 1968 sleep phases were

\footnotetext{
${ }^{1}$ Digital Sports Group, Pattern Recognition Lab, Department of Computer Science, Friedrich-Alexander-University Erlangen-Nuremberg, Erlangen, Germany.

${ }^{2}$ Department of Psychiatry and Psychotherapy, University Hospital Erlangen, Erlangen, Germany.

Corresponding author: B. Eskofier: eskofier@cs.fau.de
}

differentiated in six sleep stages according to the recommendations of Rechtschaffen \& Kales (R\&K) [5]. In 2007 the AASM Manual [6] was published as a new recommendation for sleep analysis. It categorizes sleep in only five stages: Wakefulness (W), REM-sleep (R), and three stages for nonREM sleep (N1, N2, N3). The duration and alternation of these different sleep phases during a single night is typically described in a hypnogram (Fig. 1).

Sleep analysis, i.e. the recording of the hypnogram, is usually performed by the gold standard method polysomnography (PSG) [6]. The minimum requirements for routine PSG include a total of 12 electrodes (as well as various other sensors): four for electroencephalography (EEG), three for electrooculography (EOG), three for chin electromyography (EMG), and two for electrocardiography (ECG) [6]. This is very obtrusive for the subject and may influence his normal sleep pattern. Additionally, PSG measurements can only be performed in dedicated sleep laboratories. This implies that the patient has to stay overnight and sleep in an unfamiliar environment, which might cause changes in normal sleep behavior. In consequence of these drawbacks, often more than one night is required to get reliable data.

To avoid the drawbacks of PSG many attempts have been made to find alternate methods for sleep analysis outside the sleep laboratories [7], [8], [9]. In 2007, Choi et al. assessed the feasibility of unobtrusive actigraphy using load-sensing cells integrated into the bed posts [8]. They showed that sleep/wake assessment can be done only by analyzing body movements. Also in 2007, Fox et al. compared a reference actimeter to their recording method using a Doppler radar positioned about one meter away from the subject's bed [9].

Given the high prevalence of sleep disorders in the general population, an easy sleep stage analysis at home is necessary. Users could then obtain hints about the presence of sleep disorders and have reason to go to a sleep laboratory. Although some attempts were suggested, none of these enabled the measurement of individual sleep phases without any specialized sensor.

Recently smart phones have been suggested for somnography at home. Such phones are readily available to more than $85 \%$ [10] of the general population, even in third world countries [11]. Some sleep-related applications have been presented using the built-in accelerometer of the phone to analyze body motion during sleep. However, the used algorithms are often unknown and they lack thorough scientific 
evaluation.

The purpose of this work was to provide a simple and easy to use method for preventive sleep analysis using unobtrusive motion sensors. The main contributions of this paper are as follows. Firstly, we developed an algorithm that can analyze body movements from a single accelerometer on top of the mattress and used this information to classify sleep stages into Wakefulness, REM, and non-REM. Secondly, we evaluated the algorithm on 10 subjects by comparing a reference PSG to the output of the sensor-based algorithm. Thirdly, we implemented the algorithm on a mobile phone to provide a cheap realization that is easily accessible at home.

\section{METHODS}

\section{A. Measurement devices}

Reference PSG was acquired using the SOMNOwatch plus (SOMNOmedics GmbH, Randersacker, Germany), which is a mobile and modular sleep recording device that resembles a wrist-watch. It contains a 12 bit analog/digital converter, a Li-ion battery, $64 \mathrm{MB}$ internal data storage, a triaxial accelerometer, and an ambient light sensor. The Schlaf/EEG sensor module (SOMNOmedics $\mathrm{GmbH}$ ) was additionally connected to allow recording of a single channel EEG.

To record body motion in a consistent, reliable and repeatable fashion, small wireless sensors from the Shimmer platform (Shimmer Research Ltd., Dublin, Ireland) were used. Each sensor contains a low-power microcontroller, a tri-axial accelerometer, and a Li-ion battery [12]. Accelerometer data were recorded during the whole night at a sampling rate of $204.8 \mathrm{~Hz}$ and stored on an SD-card.

\section{B. Recording procedure}

Motion activity and reference PSG were recorded during sleep for ten healthy male subjects (age 30.2 \pm 2.2 years, body mass index (BMI) $24.2 \pm 4.4 \mathrm{~kg} / \mathrm{m}^{2}$, mean \pm standard deviation (SD) ). Informed consent was obtained for all participants of the study. The recording was conducted in the home environment during two consecutive nights for each subject. This was done to reduce the influence of the first-night effect and to estimate the impact of varying environmental conditions, e.g. different mattress properties between subjects, on the measurements.

Recording was performed using the SOMNOwatch and two Shimmer sensors. One Shimmer was placed centered under the subject's pillow, the other one was placed at the foot end of the bed. Both Shimmer sensors were placed on top of the mattress without direct body contact. They were placed beneath the topmost bedsheet to avoid sensor movement. The time offsets (in seconds) between the start of each Shimmer sensor and the SOMNOwatch were recorded for every night and allowed signal synchronization. The duration of each night's record varied between six and nine hours.

Data recorded with the SOMNOwatch were preprocessed with the DOMINOlight software (SOMNOmedics $\mathrm{GmbH}$ ) and then used by a medical expert to extract sleep stages in non-overlapping 30-second epochs which were stored as reference hypnograms.

\section{Sleep stage extraction}

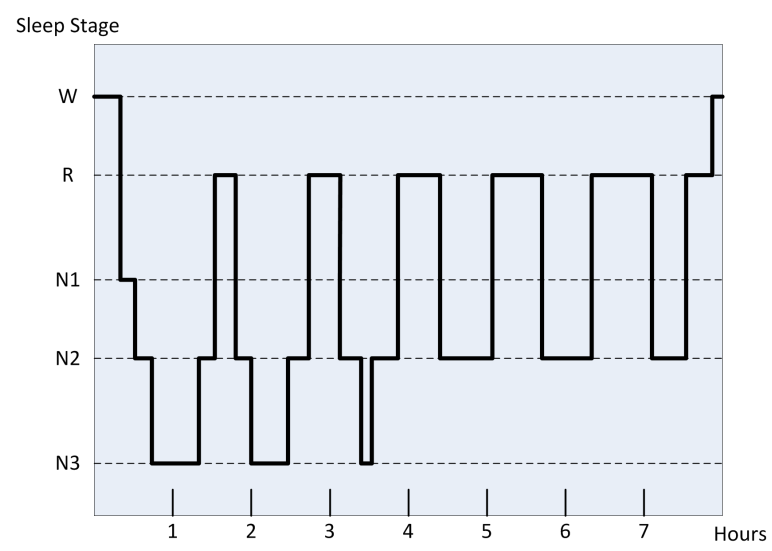

Fig. 1. Hypnogram with the different sleep stages (wakefulness (W), REMsleep (R), and non-REM sleep (N1, N2, N3)) over 8 hours of sleep.

1) Preprocessing: The raw three-axis accelerometer data were converted into SI units $\left(\mathrm{m} \mathrm{s}^{-2}\right)$ by a calibration procedure. The three signals were then passed through four digital filter operations for noise rejection and for amplification of major body movements. The operations were, in order of application:

(i) A second order low-pass filter with a cutoff frequency of about $18 \mathrm{~Hz}$ was applied. Its purpose was the rejection of high-frequency noise. The transfer function was

$$
H(z)=\frac{0.0625+0.125 z^{-1}+0.0625 z^{-2}}{1+1.3 z^{-1}-0.5 z^{-2}} .
$$

(ii) A second order derivative operation was applied to remove baseline wander and gravity components. Its transfer function was

$$
H(z)=\frac{1}{T^{2}}\left[1-z^{-1}\right]^{2}
$$

(iii) The three axis-dependent signals were aggregated to a single (axis-independent) signal by calculating the absolute sum. This reduced computational effort in further processing steps and increased the amplitude resolution of the recorded body movements. Additionally it increased overall robustness and ease of use of the method, as the algorithm becomes independent on the orientation of the motion sensor on the mattress at the beginning or during sleep.

(iv) Two separate integration operations were applied over a two second $\left(I_{2}\right)$ and four second $\left(I_{4}\right)$ window. The two resulting signals had different response characteristics to different periods of movement activity. Digital integration was chosen as it is better suited for movement identification than zero crossing methods or time above threshold [9].

2) Feature extraction: Feature extraction was based on the calculation of the body movement index [13]. For each 30-second epoch $e$ a movement index $M(e)$ was calculated from the integration output's samples with

$$
M(e)=\sum^{e}\left(I_{2} / I_{4}\right)^{2}
$$


As feature the mean over a window of 18 subsequent epochs of this body movement index was determined for each epoch. A single feature was used to allow assessment of an algorithm with minimal computational complexity. Such an algorithm could then be implemented even on very restricted mobile device hardware.

3) Classification and Evaluation: The feature computed from the Shimmer motion signals were used to build Naive Bayes classifiers that distinguished REM and non-REM sleep. For each epoch the sleep stages from the reference hypnogram were used as class label to train and evaluate the classifier.

The mean classification accuracy and its standard deviation were estimated using an exhaustive leave-one-subject-out cross-validation procedure [14]. Independent iterations of the procedure were performed with subsets including every possible night and only the second night of every subject. All iterations also contained separate evaluations for the two Shimmer sensor locations.

\section{IMPLEMENTATION}

The algorithms were initially developed and evaluated in Matlab (MathWorks Inc., Natick, USA). An additional software was developed for Android-based mobile phones using Java $^{\mathrm{TM}}$, the Android SDK 2.3.3 (Google Inc., Mountain View, USA), and the Eclipse software (Eclipse Foundation Inc., Ottawa, Canada). The Android operating system (OS) was chosen because of its open nature, widespread use, and the portability of the Java-based program code. The software consisted of a recording and a processing module.

The recording module acquired and stored motion signals in a consistent and repeatable way across any Android-based mobile device. It used the Android Sensor API ${ }^{1}$ to record the triaxial acceleration signal. Due to limitations of the OS, motion signals can only be recorded at a variable sampling rate that depended on the phone's hardware. The sampling rate was set to SENSOR_DELAY_UI. The implementation was optimized with respect to computational requirements to minimize its impact on the variation in the sampling rate. The processing module provided a minimal implementation of the classification system for mobile devices. Signal samples buffered by the recording module were used to perform the preprocessing steps of the sleep stage extraction in quasi-realtime while recording was active and some of the remaining processing steps after recording was deactivated.

To test the developed application and to assess the applicability of the proposed method two different mobile phones (Samsung GT-19000 and Samsung GT-N7000) were used for development and tests. Both used Android 2.3.6 (Google Inc.) as OS, but they were equipped with different tri-axial acceleration sensors. The mean sampling rate was $37 \mathrm{~Hz}$.

The interface of the Android application after recording and processing is shown in Fig. 2.

\footnotetext{
${ }^{1}$ http: // developer.android.com/guide/topics / sensors/sensors_motion.html
}



Fig. 2. Interface of the Android application after recording of a subject's sleep. It shows estimated/interpolated sleep stage progression.

TABLE I

MEAN CLASSIFICATION ACCURACY (WAKE VS. REM VS. NON-REM)

\begin{tabular}{|c|c|c|c|c|c|}
\hline Subject & 1 & 2 & 3 & 4 & 5 \\
\hline Accuracy [\%] & 93.9 & 64.7 & 76.7 & 65.7 & 70.6 \\
\hline
\end{tabular}

\begin{tabular}{|c|c|c|c|c|c|}
\hline Subject & 6 & 7 & 8 & 9 & 10 \\
\hline Accuracy [\%] & 78.7 & 93.2 & 86.2 & 79.7 & 83.6 \\
\hline
\end{tabular}

\section{RESULTS}

A total of 17920 epochs from the reference hypnograms of all nights were available for training and evaluation, $14.4 \%$ of those epochs were REM-epochs. Results for classification of REM, non-REM (sleep stages N1, N2, N3), and W phases are given in Tab. I as per-subject averaged classification accuracy of the Naive Bayes classifier. Averaged over all subjects, the classifier had a mean accuracy of $79.0 \%$ (SD $9.2 \%)$. Mean classification accuracy varied only slightly between data recorded at the foot of the bed and under the pillow, therefore only results from the sensor at the foot of the bed are presented.

Iterations for subsets of the second night only and persubject subsets (using both nights of a subject for crossvalidation instead of one night for validation and the other night as additional training data) had slightly worse results during the leave-one-out cross-validation. Distinguishing all five sleep stages as individual classes also yielded poor results ( $40 \%$ to $55 \%$ mean accuracy).

The classification module implemented on a mobile phone was able to perform the preprocessing steps in quasi realtime with no significant impact on the sampling rate. All operations of the algorithm could be performed on mobile device hardware. The mean sampling rate of $37 \mathrm{~Hz}$ was the highest rate that could be maintained on both phones with minimal variability.

\section{DISCUSSION}

The results showed a good agreement of $79.0 \%$ between the Naive Bayes classifier and reference hypnograms when discriminating REM, non-REM and $\mathrm{W}$ stages only. This is comparable to the results of [9], where a sensitivity and specificity of $79 \%$ and $75 \%$ were obtained analyzing 20 subjects. However, a direct comparison to previous work is difficult. Often only results for sleep/wake discrimination 
are provided or the obsolete R\&K standard is used. Also, the device used for reference measurements in this work did not fulfill the requirements for routine PSG. Yet, it can be assumed that individual sleep stages can indeed be discriminated using the proposed method.

The high standard deviation of $9.2 \%$ and the inhomogeneous results across subjects showed that the algorithm worked well for some subjects, but worse for others. This indicates that a bigger population might be needed to get consistent results. Additionally all tested subjects were healthy and between 27 and 35 years old. Further evaluation is necessary to show how the algorithm performs for older and more obese subjects.

The one described feature was based on the mean activity (i.e. a body movement index). It needs to be examined whether there are more reliable features based on different movement characteristics and if a combination of additional features might improve classification rate. Also more sophisticated classifiers, e.g. the Support Vector Machine could be used.

One possible explanation for some of the misclassifications might be the used method to extract reference hypnograms. The SOMNOwatch provided only a single channel EEG signal, which might sometimes be insufficient. Additionally no EOG was recorded, which is normally required to identify REM-stages [6]. However one goal of this work was to reduce the required effort for the subjects and the lack of additional sensors may have reduced the influence of the first-night effect.

Another important factor when analyzing sleep using body motions are the partners of the subjects. Seven of the ten male subjects slept in the same bed as their partner during the recording procedure. Three of those seven did not have separate mattresses for each person. Hence movement of the partner might have interfered with the analysis.

Future research also needs to consider mattress characteristics. Particularly, the hardness of the mattress was not examined in this work but directly affects signal characteristics of the motion activity signal initially used for preprocessing. This may cause algorithms and filters to produce different results depending on the hardness of a mattress and sensor locations.

Evaluation on the two Android-based mobile phones showed that the algorithm can be executed in real-time and that the devices are suited for unobtrusive somnography. So far no verification was performed between the mobile phones and reference PSG. This important step must be performed in future work to ensure that the mobile phone can achieve the same classification rates.

Both mobile phones were based on the Android OS, which has a task-based architecture. Consequently, motion signals of the integrated hardware sensors could only be recorded at very restricted, non-constant sampling rates. Although this did not affect the presented classifier, it is a limiting factor for the implementation of more sophisticated algorithms like ballistocardiography [15]. However, as Android is an open platform, this might change in the future.

\section{CONCLUSION}

This work proposed an algorithm for unobtrusive somnography and assessed how well individual sleep stages could be discriminated based only on body movement recorded indirectly with regular motion sensors on top of the mattress. Results showed a good agreement with reference measurements and that such an approach is feasible for use on modern mobile phones. In future this will allow creation of simple and easy to use methods for preventive sleep analysis at home.

\section{ACKNOWLEDGMENT}

This work was created in collaboration with the Embedded Systems Institute (ESI) Erlangen, supported in part by the Bavarian Ministry for Economic Affairs, Infrastructure, Transport and Technology and the European Fund for Regional Development. All trademarks are the property of their respective owners. We thank all participants.

\section{REFERENCES}

[1] C. B. Saper, G. Cano, and T. E. Scammell, "Homeostatic, circadian, and emotional regulation of sleep," The Journal of Comparative Neurology, vol. 493, no. 1, pp. 92-98, 2005.

[2] M. Reite et al., Concise Guide to Evaluation and Management of Sleep Disorders. American Psychiatric Publishing, Inc., 2002, vol. 29, no. 3.

[3] K. M. de Almondes, N. B. Mota, and J. F. Arajo, "Sleep-wake cycle pattern, sleep quality and complaints about sleep disturbances made by inpatients," Sleep science, vol. 1, pp. 36-39, 2008.

[4] Y. Harrison and J. A. Horne, "The impact of sleep deprivation on decision making: A review," Journal of Experimental Psychology: Applied, vol. 6, no. 3, pp. 236-249, 2000.

[5] A. Rechtschaffen and A. Kales, "A manual of standardized terminology, techniques and scoring system for sleep stages of human subjects," U.S. Department of Health, Education, and Welfare, Public Health Service NIH/NIND, 1968.

[6] C. Iber et al., The AASM Manual for the Scoring of Sleep and Associated Events: Rules, Terminology and Technical Specifications. American Academy of Sleep Medicine, 2007.

[7] Y. Kurihara, K. Watanabe, and H. Tanaka, "Sleep-states-transition model by body movement and estimation of sleep-stage-appearance probabilities by kalman filter," IEEE Transactions on Information Technology in Biomedicine, vol. 14(6), pp. 1428-1435, 2010.

[8] B. H. Choi, J. W. Seo, J. M. Choi, H. B. Shin, J. Y. Lee, D. U. Jeong, and K. S. Park, "Non-constraining sleep/wake monitoring system using bed actigraphy," Medical \& Biological Engineering \& Computing, vol. 45, pp. 107-114, 2007.

[9] N. A. Fox, C. Heneghan, M. Gonzalez, R. B. Shouldice, and P. de Chazal, "An evaluation of a non-contact biomotion sensor with actimetry," Conf Proc IEEE Eng Med Biol Soc, vol. 2007, pp. 26642668, 2007.

[10] Associated Press, "UN says world has 6 billion cell phone subscribers," 2012. [Online]. Available: http://news.yahoo.com/ un-says-world-6-billion-cell-phone-subscribers-111442994--finance. html

[11] I. Silva, G. B. Moody, and L. Celi, "Improving the quality of ecgs collected using mobile phones: The physionet/computing in cardiology challenge 2011," in Proc. of Computing in Cardiology, 2011.

[12] A. Burns, B. R. Greene, M. J. McGrath, T. J. O'Shea, B. Kuris, S. M. Ayer, F. Stroiescu, and V. Cionca, "Shimmer - a wireless sensor platform for noninvasive biomedical research," Sensors Journal, IEEE, vol. 10(9), pp. 1527-1534, 2010.

[13] T. Watanabe and K. Watanabe, "Noncontact method for sleep stage estimation." IEEE transactions on bio-medical engineering, vol. 51, no. 10, pp. 1735-1748, 2004.

[14] R. M. Rangayyan, Biomedical signal analysis: a case study approach. New York, NY: Wiley-Interscience [u.a.], 2002.

[15] M. Brink, C. H. Müller, and C. Schierz, "Contact-free measurement of heart rate, respiration rate, and body movements during sleep." Behavior research methods, vol. 38, no. 3, pp. 511-21, 2006. 